CERN-TH.6285/91

\title{
ANALYSIS OF ALL DIMENSIONFUL PARAMETERS RELEVANT IN GRAVITATIONAL DRESSING OF CONFORMAL THEORIES
}

\author{
H. Dorn 円 \\ Theory Division, CERN, CH-1211 Geneva 23, Switzerland \\ H.J. Otto \\ Institut für Elementarteilchenphysik, Humboldt-Universität, \\ Invalidenstr.110, D-O-1040 Berlin, Germany
}

\begin{abstract}
Starting from a covariant and background independent definition of normal ordered vertex operators we give an alternative derivation of the KPZ relation between conformal dimensions and their gravitational dressed partners. With our method we are able to study for arbitrary genus the dependence of N-point functions on all dimensionful parameters. Implications for the interpretation of gravitational dressed dimensions are discussed.
\end{abstract}

CERN-TH.6285/91

\footnotetext{
${ }^{1}$ Permanent address: IEP, Humboldt Universität, Berlin
} 
November 1991 


\section{Introduction}

If a two dimensional conformal theory, specified by the central charge $c$, the conformal dimensions $\Delta_{i}^{(0)}$ of its primary fields and the operator product expansion coefficients, is coupled to $2 \mathrm{D}$ gravity the fields get new scaling dimensions $\Delta_{i}$ which obey the KPZ relation [1, 2, 3]

$$
\Delta_{i}-\Delta_{i}^{(0)}=\frac{(\sqrt{1-c}-\sqrt{25-c})^{2}}{24} \Delta_{i}\left(1-\Delta_{i}\right) .
$$

The original derivation [1] is based on the use of the light cone gauge and $\Delta_{i}$ is related to scaling in the coordinate $z^{-}$. In the more familiar conformal gauge any relation between scaling in geometrical length and scaling in coordinates is lost after quantization of the metrics. Here the dimensions $\Delta_{i}$ are defined [2, 3] via the area dependence of N-point functions of the so called gravitational dressed primary fields, calculated for fixed area $A$ and vanishing Liouville mass (cosmological constant) $m^{2}=0$

$$
\frac{\left\langle\prod_{i}\left(V_{i}\left(z_{i}\right)\right)_{d r e s s e d}\right\rangle_{A, m^{2}=0}}{Z_{A, m^{2}=0}} \propto A^{\sum_{i}\left(1-\Delta_{i}\right)} .
$$

$Z_{A, m^{2}=0}$ is the corresponding partition function. In refs. [2, 3] the system is treated as a conformal theory in a background metrics $\hat{g}_{a b}(z)$ and the characteristic parameters as the string susceptibility and the exponents of the dressing factors are fixed by the requirement of background independence of both $Z_{A, m^{2}=0}$ and the integrated primary fields. All manipulations are performed with the formal unregularized functional integral.

The two point function for instance behaves itself by construction as a function of its coordinates like $\left|z_{1}-z_{2}\right|^{-4}$ and as a function of $A$ like $A^{2-2 \Delta}$. Since (at least if the fields are represented by scalar vertex operators) the function under discussion has naive 2D dimension zero, there must be a further dimensionful parameter involved. This is of course a renormalization scale $\mu$ originating from the suppression of self-contractions. The two point function then must look like $\left(\mu\left|z_{1}-z_{2}\right|\right)^{-4}\left(\mu^{2} A\right)^{2-2 \Delta}$. In a previous paper [4] we have given an alternative derivation of the KPZ relation based on the use of an a priori background independent and general covariant definition of normal ordered vertex operators. This procedure contains $\mu$ from the very beginning. Furthermore, we found an additional dimensional quantity $\hat{R}_{0}$ to be relevant. It parametrizes the integration constant in the Liouville action. The analysis was restricted to genus zero and vanishing cosmological constant $m^{2}=0$. Using the technique of formal continuation in the central charge, introduced in [5] and proved to be correct in [6], the aim of the present paper is a complete discussion of the dependence of $\mathrm{N}$-point functions on all dimensionful parameters for arbitrary genus of the 2D surface. The parameters are: $l$ denoting the length scale in coordinate space, $\hat{R}_{0}, \mu$ and $m^{2}$ ( $A$ is the Laplace transformed variable to $m^{2}$ ). 


\section{Analysis for genus $h \geq 2$}

We consider correlation functions of vertex operators

$$
V_{k}(z)=e^{i k X(z)}
$$

in a matter theory $\left(X^{\mu}, \mu=1, \ldots, d\right)$ described by the action

$$
S=\frac{1}{8 \pi} \int d^{2} z \sqrt{g(z)}\left(g^{m n}(z) \partial_{m} X^{\mu}(z) \partial_{n} X_{\mu}(z)+i R(z) P^{\mu} X_{\mu}(z)\right),
$$

where $R$ is the curvature scalar derived from the metrics $g_{a b}$. The background charges $P^{\mu}$ have been introduced to describe both noncritical strings $(P=0, d \neq 26)$ and minimal conformal theories $(P \neq 0, d=1)$. The central charge is

$$
c=d-3 P^{2} \text {. }
$$

After performing the $X^{\mu}$ and ghost integrations we arrive at

$$
\begin{aligned}
\left\langle\prod_{j=1}^{N} V_{k_{j}}\left(z_{j}\right)\right\rangle & =\delta\left(\sum_{j} k_{j}-P(1-h)\right) \int d \mu(\tau) \\
& \times \int \mathcal{D} \sigma e^{-(26-d) S_{L}\left[e^{\sigma} g_{a b}^{(\tau)}\right]} \\
& \times \exp \left[-\frac{1}{2} \sum_{i, j} k_{i} k_{j} G\left(z_{i}, z_{j} \mid e^{\sigma} g_{a b}^{(\tau)}\right)\right]
\end{aligned}
$$

$d \mu(\tau)$ denotes the moduli integration measure, $g_{a b}^{(\tau)}$ is a metrics completely determined by the moduli up to diffeomorphisms. $S_{L}\left[g_{a b}\right]$ is the Liouville action. Its change for $g_{a b} \rightarrow e^{\sigma} g_{a b}$ is given by the integrated conformal anomaly

$$
\begin{aligned}
S_{L}[\sigma \mid g] & \equiv S_{L}\left[e^{\sigma} g\right]-S_{L}[g] \\
& =\frac{1}{48 \pi} \int d^{2} z \sqrt{g}\left(\frac{1}{2} g^{m n} \partial_{m} \sigma \partial_{n} \sigma+R \sigma+m^{2}\left(e^{\sigma}-1\right)\right) .
\end{aligned}
$$

$G$ is the Arakelov Green function [7] which obeys

$$
G\left(z_{i}, z_{j} \mid e^{\sigma} g\right)=G\left(z_{i}, z_{j} \mid g\right)-\frac{\sigma\left(z_{i}\right)+\sigma\left(z_{j}\right)}{2(1-h)}+\frac{6}{(1-h)^{2}} S_{L}^{0}[\sigma \mid g]
$$

where $S_{L}^{0}[\sigma \mid g]$ is given by $(7)$ with $m^{2}=0$.

The Liouville field $\sigma$ is not a scalar in the present parametrization since $g_{a b}^{(\tau)}$ depends on the moduli only. Therefore, it is more convenient to choose an arbitrary reference $\hat{\sigma}$ and to parametrize the metrics with a scalar $\sigma$ by

$$
g_{a b}(z)=e^{\sigma(z)+\hat{\sigma}(z)} g_{a b}^{(\tau)}(z) \equiv e^{\sigma} \hat{g}_{a b} .
$$

The integration measure $\mathcal{D} \sigma$ is not translation invariant [8]. Using [4], [9]

$$
\mathcal{D}(\sigma+\hat{\sigma})=D_{\hat{g}} \sigma e^{S_{L}[\sigma \mid \hat{g}]}
$$


with translation invariant $D_{\hat{g}} \sigma$ we get from (6)

$$
\begin{aligned}
\left\langle\prod_{j=1}^{N} V_{k_{j}}\left(z_{j}\right)\right\rangle & =\delta\left(\sum_{j} k_{j}-P(1-h)\right) \int d \mu(\tau) e^{-(26-d) S_{L}[\hat{g}]} \\
& \times \int D_{\hat{g}} \sigma e^{-(25-d) S_{L}[\sigma \mid \hat{g}]} \exp \left[-\frac{1}{2} \sum_{i, j} k_{i} k_{j} G\left(z_{i}, z_{j} \mid e^{\sigma} \hat{g}\right)\right] .
\end{aligned}
$$

For the discussion of the ultraviolet regularization we split

$$
G\left(z_{i}, z_{j} \mid g\right)=-\log \left(M^{2}\left|z_{i}-z_{j}\right|^{2}\right)+G_{M}\left(z_{i}, z_{j} \mid g\right)
$$

$\mathrm{M}$ is an auxiliary scale. It will be related below in a convenient way to the parameters mentioned in the introduction. $G_{M}$, defined by (12), is regular at $z_{i}=z_{j}$ and obeys the same scaling relation (8) as $\mathrm{G}$ itself.

We now regularize the logarithm as

$$
\log \left(M^{2}\left|z_{i}-z_{j}\right|^{2}\right) \longrightarrow \log \left(M^{2}\left[\left|z_{i}-z_{j}\right|^{2}+\epsilon^{2} / \mu^{2}\right]\right)
$$

with dimensionless $\epsilon$ and RG-mass scale $\mu$. Furthermore, the normal product version of $V_{k}(z)$ is defined [4] by multiplication with a general covariant factor whose single purpose is the cancellation of the $\epsilon$ singularities after quantization of both $X^{\mu}$ and $\sigma$

$$
: V_{k}(z):=V_{k}(z)\left(\epsilon^{2} e^{\sigma} \sqrt{\hat{g}}\right)^{a(k)-1} e^{\sigma} \sqrt{\hat{g}}
$$

The last factor $e^{\sigma} \sqrt{\hat{g}}=\sqrt{g}$ is added since after quantization of the metrics only scalar densities make sense. With the technique used below the Liouville interaction term $m^{2} e^{\sigma}$ appears as an operator insertion evaluated with $S_{L}^{0}$. To cancel also the ultraviolet singularities of this operator we finally define as our regularized starting point

$$
\begin{aligned}
\left\langle\prod_{j=1}^{N}: V_{k_{j}}\left(z_{j}\right):\right\rangle & =\delta\left(\sum_{j} k_{j}-P(1-h)\right) \int d \mu(\tau) e^{-(26-d) S_{L}[\hat{g}]} e^{\frac{m^{2} Q^{2} \hat{A}}{16 \pi}} \\
& \times \prod_{j}\left(\left(\frac{M}{\mu}\right)^{k_{j}^{2}} \epsilon^{k_{j}^{2}+2\left(a_{j}-1\right)}\left(\hat{g}\left(z_{j}\right)\right)^{\frac{a_{j}}{2}}\right) \prod_{i \neq j}\left(M\left|z_{i}-z_{j}\right|\right)^{k_{i} k_{j}} \\
& \left.\times \int_{\hat{g}} \sigma e^{-3 Q^{2}\left(S_{L}^{0}[\sigma \mid \hat{g}]+\frac{m^{2}}{48 \pi} \epsilon^{2\left(a_{0}-1\right)} \int d^{2} z \hat{g} \frac{a_{0}}{2}\right.} e^{a_{0} \sigma}\right) \\
& \times \prod_{j} e^{a_{j} \sigma\left(z_{j}\right)} \exp \left[-\frac{1}{2} \sum_{i, j} k_{i} k_{j} G_{M}\left(z_{i}, z_{j} \mid e^{\sigma} \hat{g}\right)\right],
\end{aligned}
$$

with $\hat{A}$ being the area corresponding to $\hat{g}$,

$$
Q^{2}=\frac{25-d}{3}
$$

and

$$
a_{i}=a\left(k_{i}\right), \quad a_{0}=a(0) .
$$


Using the scaling relation (8) for $G_{M}$ and taking into account the $\delta$-function constraint, the $\sigma$-functional integral in (15) becomes

$$
\begin{aligned}
I & =\exp \left[-\frac{1}{2} \sum_{i, j} k_{i} k_{j} G_{M}\left(z_{i}, z_{j} \mid \hat{g}\right)\right] \int D_{\hat{g}} \sigma \prod_{j=1}^{N} e^{\left(a_{j}+\frac{P k_{j}}{2}\right) \sigma\left(z_{j}\right)} \\
& \times e^{-3\left(Q^{2}+P^{2}\right) S_{L}^{0}[\sigma \mid \hat{g}]} \exp \left[-\frac{m^{2} Q^{2}}{16 \pi} \epsilon^{2\left(a_{0}-1\right)} \int d^{2} z \hat{g}^{\frac{a_{0}}{2}} e^{a_{0} \sigma}\right] .
\end{aligned}
$$

Now we perform the $\sigma$ integration à la ref. [5] with the zero mode defined appropriately for the use of the Arakelov Green function $\left(\sigma_{0}=(8 \pi(1-h))^{-1} \int d^{2} z \sqrt{\hat{g}} \hat{R} \sigma\right)$. For abbreviation we introduce

$$
b_{i}=a_{i}+\frac{P k_{i}}{2}
$$

and

$$
s=\frac{\frac{Q^{2}}{2}(1-h)-\sum_{i} a_{i}}{a_{0}}=\frac{\frac{Q^{2}+P^{2}}{2}(1-h)-\sum_{i} b_{i}}{a_{0}} .
$$

As usual we start with positive integer s and continue analytically afterwards. From now on capital indices $I, J, \ldots$ will be understood as running between 1 and $s$, while $i, j, \ldots$ refer to the vertex insertion points $1,2, \ldots, N$ as before.

$$
\begin{aligned}
I & =\left|a_{0}\right|^{-1} \Gamma(-s)\left(\frac{Q^{2} m^{2}}{16 \pi}\right)^{s} \epsilon^{2 s\left(a_{0}-1\right)} e^{S_{L}[\hat{g}]} \\
& \times\left(\frac{M \epsilon}{\mu}\right)^{-4 \frac{\sum_{i} b_{i}^{2}}{Q^{2}+P^{2}}-4 \frac{s a_{0}^{2}}{Q^{2}+P^{2}}} \prod_{i \neq j}\left(M\left|z_{i}-z_{j}\right|\right)^{-4 \frac{b_{i} b_{j}}{Q^{2}+P^{2}}} \\
& \times \quad \int \prod_{J}\left(d^{2} w_{J} \hat{g}\left(w_{J}\right)^{\frac{a_{0}}{2}}\right) \prod_{I \neq J}\left(M\left|w_{I}-w_{J}\right|\right)^{-4 \frac{a_{0}^{2}}{Q^{2}+P^{2}}} \prod_{i, J}\left(M\left|z_{i}-w_{J}\right|\right)^{-8 \frac{a_{0} b_{i}}{Q^{2}+P^{2}}} \\
& \times \quad \exp \left[\sum_{i, j}\left(-\frac{1}{2} k_{i} k_{j}+\frac{2}{Q^{2}+P^{2}} b_{i} b_{j}\right) G_{M}\left(z_{i}, z_{j} \mid \hat{g}\right)\right. \\
& \left.\quad+\frac{2}{Q^{2}+P^{2}}\left(\sum_{I, J} a_{0}^{2} G_{M}\left(w_{I}, w_{J} \mid \hat{g}\right)+2 \sum_{i, J} a_{0} b_{i} G_{M}\left(z_{i}, w_{J} \mid \hat{g}\right)\right)\right] .
\end{aligned}
$$

Putting the integral $I$ back into (15) we find cancellation of $\epsilon$ for

$$
k_{i}^{2}+2\left(a_{i}-1\right)-4 \frac{b_{i}^{2}}{Q^{2}+P^{2}}=0
$$

which means

$$
\begin{gathered}
b_{i}=\frac{Q^{2}+P^{2}}{4}\left(1 \pm \sqrt{1+\frac{8}{Q^{2}+P^{2}}\left(\Delta_{0}\left(k_{i}\right)-1\right)}\right) \\
a_{0}=\frac{Q^{2}+P^{2}}{4}\left(1-\sqrt{1-\frac{8}{Q^{2}+P^{2}}}\right) .
\end{gathered}
$$

$\Delta_{0}(k)$ is the conformal dimension of the original $V_{k}(z)$

$$
\Delta_{0}(k)=\frac{k(k-P)}{2} .
$$


As usual the sign ambiguity for $a_{0}$ has been resolved by correspondence with the quasiclassical limit [3] or by requiring microscopic nature of $e^{a_{0} \sigma}$ [10]. For comparison with other treatments we note that our Liouville field is normalized just as it appears in the conformal anomaly of the $X$ integration. To compare e.g. with ref. [3] one has to rescale $\sigma \rightarrow-2 \sigma / \sqrt{Q^{2}+P^{2}}$. Altogether, using (22) we get from (15)

$$
\begin{aligned}
\left\langle\prod_{j}: V_{k_{j}}\left(z_{j}\right):\right\rangle & =\delta\left(\sum_{j} k_{j}-P(1-h)\right) \int d \mu(\tau) e^{-3 Q^{2} S_{L}^{0}[\hat{g}]}\left|a_{0}\right|^{-1} \Gamma(-s) \\
& \times\left(\frac{Q^{2} m^{2}}{16 \pi}\right)^{s} \prod_{j}\left(\hat{g}\left(z_{j}\right)\right)^{\frac{a_{j}}{2}}\left(\frac{M}{\mu}\right)^{-\sum_{i} 2\left(a_{i}-1\right)-2 s\left(a_{0}-1\right)} \\
& \times \prod_{i \neq j}\left(M\left|z_{i}-z_{j}\right|\right)^{k_{i} k_{j}-4 \frac{b_{i} b_{j}}{Q^{2}+P^{2}}} \int \prod_{J}\left(d^{2} w_{J} \hat{g}\left(w_{J}\right)^{\frac{a_{0}}{2}}\right) \\
& \times \prod_{I \neq J}\left(M\left|w_{I}-w_{J}\right|\right)^{\frac{-4 a_{0}^{2}}{Q^{2}+P^{2}}} \prod_{i, J}\left(M\left|z_{i}-w_{J}\right|\right)^{\frac{-8 a_{0} b_{i}}{Q^{2}+P^{2}}} J_{M}(z, w \mid \hat{g}) .
\end{aligned}
$$

The definition of $J_{M}$ is obvious from (21), it is the product of all factors containing $G_{M}$.

By construction there is no dependence on $\mathrm{M}$ and $\hat{\sigma}$. As a consistency check one can verify this for eq.(26) explictely by using the $\delta$ - constraint, (20,22). During this exercise one finds

$$
\begin{gathered}
J_{\lambda M}(z, w \mid \hat{g})=\lambda^{Q^{2}(1-h)^{2}} J_{M}(z, w \mid \hat{g}) \\
J_{M}\left(z, w \mid e^{\varphi} \hat{g}\right)=J_{M}(z, w \mid \hat{g}) e^{3 Q^{2} S_{L}^{0}[\varphi \mid \hat{g}]} \prod_{j} e^{-a_{j} \varphi\left(z_{j}\right)} \prod_{J} e^{-a_{0} \varphi\left(w_{J}\right)} .
\end{gathered}
$$

Thanks to $\hat{\sigma}$ - independence we now can choose $\hat{\sigma}$ to describe a constant curvature metrics. Let $\hat{R}$ denote the corresponding curvature scalar. Then

$$
S_{L}^{0}[\hat{g}]=S_{L}^{0}\left(\hat{R}, \tau_{i}\right)
$$

is a usual function of $\hat{R}$ and $\tau_{i}$. Specializing (7) to this situation we find

$$
\frac{d}{d \hat{R}} S_{L}^{0}\left(\hat{R}, \tau_{i}\right)=-\frac{1-h}{6 \hat{R}} .
$$

This implies

$$
S_{L}^{0}\left(\hat{R}, \tau_{i}\right)=S_{L}^{0}\left(\hat{R} / \hat{R}_{0}\right)=-\frac{1-h}{6} \log \left(\hat{R} / \hat{R}_{0}\right)
$$

with an integration constant $\propto \log \hat{R}_{0}$ which can be taken $\tau_{i}$ - independent. The moduli occur in the general case, of course,

$$
S_{L}^{0}\left[e^{\sigma} \hat{g}\right]=-\frac{1-h}{6} \log \left(\hat{R} / \hat{R}_{0}\right)+\frac{1}{48 \pi} \int d^{2} z \sqrt{\hat{g}}\left(\frac{1}{2} \hat{g}^{m n} \partial_{m} \sigma \partial_{n} \sigma+\hat{R} \sigma\right)
$$

via the boundaries of the $z$-integration in a Fuchsian or Schottky type parametrization of the surface. 
Now we recognize a $\hat{R}_{0}$-dependence of (26) via $S_{L}^{0}[\hat{g}]$. As before there is no dependence on $\hat{R}$ and $M$. Therefore, we are free to put

$$
\hat{R}=M^{2}=\hat{R}_{0}
$$

From (27) and (28) we get with (20), (22)

$$
J_{\left(\lambda \hat{R}_{0}\right)^{\frac{1}{2}}}\left(z, w \mid \lambda^{-1} \hat{g}_{0}\right)=\lambda^{\frac{Q^{2}(1-h)^{2}}{2}} J_{\hat{R}_{0}^{\frac{1}{2}}}\left(z, w \mid \hat{g}_{0}\right) .
$$

Here $\hat{g}_{0}$ denotes the metric yielding $\hat{R}_{0}$.

Now we are ready to study the scaling behaviour of (26). Concerning the coordinate dependence we are interested in uniform scaling, i.e.

$$
z_{i}=l \cdot \xi_{i}, \quad w_{I}=l \cdot \eta_{I}
$$

with dimensionless $\xi_{i}, \eta_{I}$. $J$ is dimensionless and depends on two dimensionful parameters : $\hat{R}_{0}$ and $l$. Therefore, we get from (34)

$$
J_{\hat{R}_{0}^{\frac{1}{2}}}\left(l \xi, l \eta \mid \hat{g}_{0}\right)=\left(\hat{R}_{0} l^{2}\right)^{\frac{Q^{2}(1-h)^{2}}{2}} f(\xi, \eta, \tau) .
$$

A last necessary information concerns $\hat{g}_{0}$. We use the uniformization theorem to represent our surface as the upper half plane divided by some subgroup of the isometry group of the constant curvature metrics [11]

$$
d s^{2}=\frac{-2}{\hat{R}_{0}(\operatorname{Imz})^{2}} d z d \bar{z}
$$

This implies

$$
\left(\hat{g}_{0}\left(z_{i}\right)\right)^{\frac{1}{2}}=\left(\hat{R}_{0} l^{2}\right)^{-1}\left(\hat{g}_{0}\left(\xi_{i}\right)\right)^{\frac{1}{2}}
$$

We use eqs. (33),(35),(36),(38), again the $\delta$-constraint and $(20),(22)$ and take into account that the scaling in coordinate space also affects the boundaries but does not change the dimensionless moduli. We then get finally

$$
\left\langle\prod_{j}: V_{k_{j}}\left(z_{j}\right):\right\rangle=\left[\left(\frac{\mu^{2}}{m^{2}}\right)^{2-\gamma_{0}}\left(\frac{\hat{R}_{0}}{\mu^{2}}\right)^{\frac{25-d}{6}}\right]^{h-1}\left(\frac{m}{\mu}\right)^{2 \sum_{j} \Delta_{j}}(m l)^{-2 N} F_{h, N}(\xi)
$$

with

$$
\begin{aligned}
F_{h, N}(\xi) & =\delta\left(\sum_{j} k_{j}-P(1-h)\right)\left|a_{0}\right|^{-1} \Gamma(-s)\left(\frac{Q^{2}}{16 \pi}\right)^{s} \prod_{j}\left(\hat{g}_{0}\left(\xi_{j}\right)\right)^{\frac{a_{j}}{2}} \\
& \times \prod_{i \neq j}\left|\xi_{i}-\xi_{j}\right|^{k_{i} k_{j}-4 \frac{b_{i} b_{j}}{Q^{2}+P^{2}}} \int d \mu(\tau) \int \prod_{J}\left(d^{2} \eta_{J} \hat{g}_{0}\left(\eta_{J}\right)^{\frac{a_{0}}{2}}\right) \\
& \times \prod_{I \neq J}\left|\eta_{I}-\eta_{J}\right|^{-4 \frac{a_{0}^{2}}{Q^{2}+P^{2}}} \prod_{i, J}\left|\xi_{i}-\eta_{J}\right|^{-8 \frac{a_{0} b_{i}}{Q^{2}+P^{2}}} f(\xi, \eta, \tau) .
\end{aligned}
$$

The dimensions $\Delta_{i}$ are defined by

$$
\frac{b_{i}}{a_{0}}=1-\Delta_{i}
$$

and $\gamma_{0}$ denotes the string susceptibility [2, 3]

$$
\gamma_{0}=2-\frac{Q^{2}+P^{2}}{2 a_{0}}=2+\frac{1}{12}(c-25-\sqrt{(25-c)(1-c)}) .
$$




\section{Analysis for $h=0,1$}

The sphere fits into the above analysis up to eqs. $(37,38)$. However, it is straightforward to determine $J$ explicitely

$$
J_{\hat{R}_{0}^{\frac{1}{2}}}\left(z, w \mid \hat{g}_{0}\right)=e^{\frac{Q^{2}}{2}(-1+\log 8)} \prod_{j} e^{-a_{j} \hat{\sigma}_{0}\left(z_{j}\right)} \prod_{J} e^{-a_{0} \hat{\sigma}_{0}\left(w_{J}\right)} .
$$

Then $\hat{\sigma}_{0}$ cancels and (39) remains correct for $\mathrm{h}=0$, too. (The parameter $M$ of ref. [4] is related to $\hat{R}_{0}$ by $\hat{R}_{0}=8 M^{2} e^{-1}$.)

A little bit more thought is needed for the torus $h=1$. In this case we cannot use the Arakelov Green function and are forced to exploit the more standard Green function with the zero mode defined by weighting with $\hat{g}^{\frac{1}{2}}$ instead of $\hat{g}^{\frac{1}{2}} \hat{R}$. Then in the scaling relation replacing (8) there appears the integral $\int d^{2} z G\left(z_{i}, z \mid \hat{g}\right) e^{\sigma} \sqrt{\hat{g}}$ instead of the local $\sigma\left(z_{i}\right)$ [11, 12]. But fortunately, just for $h=1$ we always have momentum conservation $\sum_{i} k_{i}=0$ and that unpleasant nonlocal quantity drops out in the $X^{\mu_{-}}$ integration. As there is no constantly curved torus there is no $\hat{R}_{0}$-dependence. For the $\sigma$ - integration we can refer to flat $\hat{g}$ and use the standard expression of $\mathrm{G}$ by Theta functions. After all we reproduce the dimensional factors in (39) with $h=1$.

\section{Discussion}

The scaling behaviour in $m, \mu, l, \hat{R}_{0}$ can be directly read off from our final result $(39),(40)$ if $F_{h, N}(\xi)$ is finite and nonzero. While in general one expects this to be true ( for a computation of $F_{0, N}$ see [5, 6]), there are certain important exceptions. The first obvious one is the case of integer $s \geq 0$. An example for such a situation is the genus one partition function $(s=0)$. The singularity is due to the divergent $\sigma$-zero mode integration used in going from eq.(18) to eq.(21). For noninteger $s>0$ the analytic continuation from $s<0$ yields finite results, but for integer $s \geq 0$ we are just sitting on the poles of the $\Gamma$-function. The $\sigma_{0}$-integral is divergent at $s \geq 0$ for $\sigma_{0} \rightarrow-\infty$. This is an ultraviolet problem in geometrical length. Therefore, our treatment of the UV-problem was not quite complete. It cancels only divergencies due to selfcontractions of vertex operators built from $X^{\mu}$ and the nonzero mode of $\sigma$. We do not know at present how to relate a cutoff in $\sigma_{0}$ [14, 15 to our $\epsilon$ in a covariant and background independent way, since the splitting in $\sigma=\sigma_{0}+\tilde{\sigma}$ always refers to a background $\hat{g}$.

Fortunately, one can proceed by an indirect argument, based on observations in [10, 16]. Since the area operator $\int d^{2} z: V_{0}$ : gives a contribution (-1) to $s$ one finds that for an arbitrary $N$ point function

$$
\left(\frac{d}{d m^{2}}\right)^{[s]+1}\left\langle\prod_{j}: V_{k_{j}}\left(z_{j}\right):\right\rangle
$$

has a total $s$-value $<0$ corresponding to a convergent $\sigma_{0}$-integration. Applying to this quantity the now unquestioned formulas $(39),(40)$ we reproduce the latter after $[s]+1$ 
integrations for the original $s \geq 0$, but with two modifications. There are additional nonuniversal (i.e. with renormalization scheme dependent coefficients) integer powers $\left(m^{2} / \mu^{2}\right)^{i} ; \mathrm{i}=0,1, \ldots,[\mathrm{s}]$ due to $[\mathrm{s}]+1$ free integration constants. For integer $\mathrm{s} \geq 0$ in the leading universal term $\left(m^{2} / \mu^{2}\right)^{s}$ is replaced by $\left(m^{2} / \mu^{2}\right)^{s} \log \left(m^{2} / \mu^{2}\right)$.

A further potential source of divergent $F_{h, N}$ is the moduli integration. Since the moduli are dimensionless a corresponding cutoff does not influence the balance of dimensionful scales in our scheme. The relation to other statements in the literature [10, 16] still has to be clarified.

A large amount of activity has been devoted to the $c=1$ case. Here $F_{h, N}$ is zero (for an explicit discussion for $h=0$ see [5, 17]). This is due to the fact that at $c=1$ there has to be made a new choice for the cosmological constant operator [10, 15].

After this digression concerning the validity and some modifications of $(39),(40)$ we now turn to the discussion of the scaling properties themselves. Formulas are always understood up to logarithmic corrections and nonuniversal power terms. With $N=0$ eq.(39) yields the scaling property of the partition function $Z_{h}$

$$
Z_{h} \propto\left[\left(\frac{\mu^{2}}{m^{2}}\right)^{2-\gamma_{0}}\left(\frac{\hat{R}_{0}}{\mu^{2}}\right)^{\frac{25-d}{6}}\right]^{h-1} .
$$

As mentioned in the introduction, the area is the only geometrical quantity left after $\sigma$-integration. As familiar from [2, 3] we can divide the $\sigma$-integration in (18) into two steps

$$
\begin{aligned}
I & =e^{-\frac{1}{2} \sum_{i, j} k_{i} k_{j} G} \int_{0}^{\infty} d A e^{-m^{2} A} \int D_{\hat{g}} \sigma \delta\left(\frac{Q^{2}}{16 \pi} \epsilon^{2\left(a_{0}-1\right)} \int d^{2} z \hat{g}^{\frac{a_{0}}{2}} e^{a_{0} \sigma}-A\right) \\
& \times \prod_{j} e^{b_{j} \sigma\left(z_{j}\right)} e^{-3\left(P^{2}+Q^{2}\right) S_{L}^{0}} .
\end{aligned}
$$

Introduced in this manner $A$ is nothing more than the Laplace transformed variable of $m^{2}$ and every scaling $\left(m^{2}\right)^{y}$ translates into $A^{-1-y}$. Besides this technical area variable A there is of course the expectation value of the area $\bar{A}$

$$
\bar{A}=\frac{\int d^{2} z\left\langle: V_{0}(z):\right\rangle}{Z_{h}} \propto m^{-2}
$$

The normalized N-point functions

$$
\frac{\left\langle\prod_{j}: V_{k_{j}}\left(z_{j}\right):\right\rangle}{Z_{h}} \propto\left(\frac{m}{\mu}\right)^{2 \sum_{j} \Delta_{j}}(m l)^{-2 N}
$$

are independent on $\hat{R}_{0}$. Since $\hat{R}_{0}$ parametrizes a field independent constant in $S_{L}$ this was clear from the beginning. However, $\hat{R}_{0}$ remains present in the sum over all genera of the integrated N-point function relevant for string applications.

$$
\frac{\sum_{h}\left\langle\prod_{j}\left(\int d^{2} z_{j}: V_{k_{j}}\left(z_{j}\right):\right)\right\rangle}{\sum_{h} Z_{h}}=\left(\frac{m}{\mu}\right)^{2 \sum_{j} \Delta_{j}}(m)^{-2 N} \frac{\sum_{h} g_{\text {eff }}^{h} H_{h, N}}{\sum_{h} g_{\text {eff }}^{h} H_{h, 0}}
$$


with $H_{h, N}=\int \prod_{i} d^{2} \xi_{i} F_{h, N}(\xi)$ and

$$
g_{e f f}=g\left(\frac{\mu^{2}}{m^{2}}\right)^{2-\gamma_{0}}\left(\frac{\hat{R}_{0}}{\mu^{2}}\right)^{\frac{25-d}{6}} .
$$

$g$ is the string coupling. The structure of $g_{\text {eff }}$ resembles that of the effective coupling in matrix models [13].

A remarkable fact about (48) is the following. The contribution of $m^{2}$ is governed by the central charge $c$, that of $\hat{R}_{0}$ by the dimension $d$ of the string target space and that of $\mu$ by both $c$ and $d$.

A last remark concerns the relation to our previous paper [4] where the $m^{2}=0$ case was treated. Requiring finite results for $m^{2} \rightarrow 0$ one has to ensure vanishing exponents of $\mathrm{m}^{2}$. This reintroduces the second $\delta$-constraint produced otherwise by the $\sigma$-zero mode integration in the massless case. Then,to get the scaling powers $\Delta_{i}$,scaling in $\hat{R}_{0}$ is crucial (" $\lambda$-scaling").

\section{Acknowledgements}

H.D. thanks the CERN Theoretical Division for kind hospitality and financial support as well as L. Alvarez-Gaumé and D. Lüst for discussions. 


\section{References}

[1] A.M. Polyakov, Mod. Phys. Lett. A2 (1987) 893

V.G. Knizhnik, A.M. Polyakov, A.B. Zamolodchikov, Mod. Phys. Lett. A3 (1988) 819

[2] F. David, Mod. Phys. Lett. A3 (1988) 1651

[3] J. Distler,H. Kawai, Nucl. Phys. B321 (1989) 509

[4] H. Dorn, H.-J. Otto, Phys. Lett. B232 (1989) 327

[5] M. Goulian, M. Li, Phys. Rev. Lett. 66 (1991) 2051

P. Di Francesco, D. Kutasov, Phys. Lett. B261(1991) 385

[6] K. Aoki, E. D’Hoker, preprint UCLA/91/TEP/32, Los Angeles 1991

[7] E. D'Hoker, D. H. Phong , Comm. Math. Phys. 124 (1989) 629

[8] K. Fujikawa, Nucl. Phys. B226 (1983) 437

[9] N. Mavromatos, J. Miramontes, Mod. Phys. Lett. A4 (1989) 1849

E. D'Hoker, P. S. Kurzepa, Mod. Phys. Lett. A5 (1990) 1411

[10] N. Seiberg,Rutgers preprint RU-90-29, Piscataway 1990

[11] E. D’Hoker, D. H. Phong, Rev. Mod. Phys. 60 (1988) 917

[12] H. Dorn, H.-J. Otto, Phys. Lett. B221 (1989) 16

[13] M. R. Douglas, S. H. Shenker, Nucl. Phys. B335 (1990) 635

[14] A. M. Polyakov, Mod. Phys. Lett. A6 (1991) 635

[15] J. Polchinski, Nucl. Phys. B346 (1990) 253

[16] J. Polchinski, Texas University preprint UTTG-19-90

[17] I. R. Klebanov, Lectures at ICTP spring school 1991, PUPT-1271 